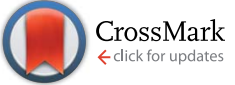

Cite this: RSC Adv., 2017, 7, 16273
Received 9th February 2017 Accepted 3rd March 2017

DOI: 10.1039/c7ra01647f

rsc.li/rsc-advances

\section{Enhanced adsorptive removal of anionic and cationic dyes from single or mixed dye solutions using MOF PCN-222 $\uparrow$}

\author{
Haichao Li, ${ }^{a}$ Xinyu Cao, ${ }^{a}$ Chuang Zhang, ${ }^{a}$ Qing Yu, ${ }^{a}$ Zijian Zhao, ${ }^{a}$ Xuedun Niu, ${ }^{b}$ \\ Xiaodong Sun, ${ }^{b}$ Yunling Liu, ${ }^{b}$ Li Ma*ac and Zhengqiang Li*a
}

\begin{abstract}
Metal-organic frameworks (MOFs) are attractive for the removal of industrial dyes from aqueous pollution. However, effective simultaneous removal of oppositely charged dye ions is still a challenge. We find that zirconium-metalloporphyrin mesoMOF (PCN-222/MOF-545) exhibits excellent adsorption/removal capacities for numerous anionic and cationic dyes individually and together in solution. PCN-222 is fabricated by solvothermal synthesis and characterized by powder XRD and FT-IR methods to confirm its structure. $\mathrm{N}_{2}$ adsorption/desorption indicates PCN-222 has a large pore size of $3.2 \mathrm{~nm}$ and a surface area of $2336 \mathrm{~m}^{2} \mathrm{~g}^{-1}$. The zeta potential measurement shows that PCN-222 has a slight basic isoelectric point at $\mathrm{pH} 8$ with appropriate potentials of 23.5 and $-13.6 \mathrm{mV}$ in the range of $\mathrm{pH} 3-10$. These features facilitate the dual-function of PCN-222 toward anionic and cationic dyes, or even their complexes. The results show that $\mathrm{PCN}-222$ has a maximum loading efficacy of $906 \mathrm{mg} \mathrm{g}^{-1}$ for anionic methylene blue (MB) and $589 \mathrm{mg} \mathrm{g}^{-1}$ for cationic methyl orange (MO) in a single dye system. Interestingly, when MB and $\mathrm{MO}$ co-exist in solution, they mutually enhance the capability by $36.8 \%$ (1239 $\mathrm{mg} \mathrm{g}^{-1}$ ) for MB and $73.5 \%$ (1022 $\mathrm{mg} \mathrm{g}^{-1}$ ) for $\mathrm{MO}$. The adsorption kinetics, isotherms, stability and reusability of PCN-222 are reported. The results reveal that no significant changes of either crystal structure or loading capacity are observed up to eight recycles during the removal of $M O$ and MB in solution. The adsorption efficacies of eight representative dyes are presented. Compared with previously published data, the capacity of PCN222 ranks at the top. We prepared a facile adsorption chromatography column to demonstrate the broad application potentials of PCN-222 in dye removal from aqueous pollution. Finally, we propose a pushpull mechanism to explain the mutual enhancement of adsorption in a mixture dye solution.
\end{abstract}

\section{Introduction}

Pollutants from the textile, paper, leather, plastic, rubber, cosmetic, and food industries can damage environmental safety, living species and global ecosystems if no adequate treatments are available. ${ }^{1,2}$ Organic dyes are widely used in various manufacturing industries and they are dangerous organic pollutants. ${ }^{3}$ About 10$15 \%$ of commercial dyes produced from the textile industries are discharged into the environment every year. ${ }^{4}$ Most of them are toxic and carcinogenic. ${ }^{5}$ Thus, removing dyes from industrial waste has become an important research topic. As we know,

${ }^{a}$ Key Laboratory for Molecular Enzymology, Engineering of Ministry of Education, School of Life Sciences, Jilin University, 2699 Qianjin Street, Changchun 130012, Jilin Province, PR China. E-mail: lzq@jlu.edu.cn; Tel: +86-431-85155201

${ }^{b}$ State Key Laboratory of Inorganic Synthesis \& Preparative Chemistry, College of Chemistry, Jilin University, 2699 Qianjin Street, Changchun 130012, Jilin Province, PR China

'Department of Physics, Georgia Southern University, Statesboro, GA 30460, USA. E-mail: Ima@georgiasouthern.edu

$\dagger$ Electronic supplementary information (ESI) available. See DOI: $10.1039 / \mathrm{c} 7 \mathrm{ra0} 01647 \mathrm{f}$ organic dyes are difficult to decompose under natural condition because of their stable and complex chemical structures. Extensive efforts have been made to eliminate dye contamination from wastewater, including physical, chemical, and biological approaches. ${ }^{6}$ Among various treatment technologies adsorption is the most attractive method owing to its simplicity and efficiency. ${ }^{7}$ The materials contain natural and synthetic adsorbents. ${ }^{8}$ In the past few years, a number of adsorption materials have been used to treat industrial wastewater, such as activated carbon, carbon nanotubes, clay, zeolites, graphene, polymeric resins and modified diatomites. ${ }^{3,9-14}$ Activated carbon is one of the most popular adsorbents because of its high porosity, strong interaction, chemical inertness and non-toxicity. However, most of their pore diameters are less than $2 \mathrm{~nm}$, so that some bulky dye molecules cannot be captured on the inner surface of the pores, which limits the utilization ratio of the relatively large surface areas. Furthermore, commercially available activated carbons may be expensive and non-renewable. ${ }^{15,16}$

Metal-organic frameworks (MOFs) are a class of crystalline material with high porosity composed of metal clusters and organic linkers. MOFs have application potential in adsorption, 
membrane separation, sensing, catalysis, and proton conduction. Recently, MOFs have been developed for use in the removal of dye contamination from waste solutions. ${ }^{17}$ Compared with other porous adsorbents, like activated carbon, zeolites or metalcomplex hydrides, MOFs have many advantages, such as ultralow density, discrete ordered structure, large specific surface area, simplicity in synthesis, and good thermal stability, which are suitable for physical and chemical applications. ${ }^{18,19}$ Furthermore, the high stability in water, large surface area and correct pore size are critical properties of MOFs in the adsorption/removal of organic pollutants. The application of MOFs for the adsorptive removal of dyes was first reported using Cr-BDCs (MIL-53 or MIL101) toward methyl orange (MO) by Jhung's group. ${ }^{20}$ This work also showed that by adding a positive group by post-synthesis modification, PED-MIL-101 increases the adsorption capacity toward $\mathrm{MO}^{20}$ The same group also later studied adsorptive removal of both methylene blue (MB) and MO by iron terephthalate MOF-235. ${ }^{21}$ Zhong's group reported that MIL-100(Fe) can removal both $\mathrm{MB}$ and MO, while MIL-100(Cr) can selectively adsorb MB. ${ }^{22}$ Recently Sun's group showed that a waterstable DUT-23(Cu) has high adsorption capacity toward MB. ${ }^{23}$

A zirconium-metalloporphyrin mesoMOF PCN-222 (MOF545) was first synthesized by Zhou and his co-workers and also independently reported by Yaghi, called MOF-545. ${ }^{24,25}$ PCN222 has a large open channel with a diameter of $3.7 \mathrm{~nm}$ while most MOFs are under $2 \mathrm{~nm}$. To support the large diameter, PCN-222 is composed of $\mathrm{Zr}_{6}$ clusters and TCPPs (tetrakis(4carboxyphenyl)porphyrin). PCN-222 is stable in aqueous solution at pH 3-10. The porphyrin frame could be metal-free, or with a series of metal ions ( $\mathrm{Mn}, \mathrm{Fe}, \mathrm{Co}, \mathrm{Ni}, \mathrm{Cu}, \mathrm{Zn}, \mathrm{Pd}$ ) for different applications, such as $\mathrm{PCN}-222(\mathrm{Fe})$, which possesses peroxidase activity, ${ }^{24}$ or $\mathrm{PCN}-222(\mathrm{Pd})$, which can serve as a fluorescent "turn on" sensor. ${ }^{26}$ The PCN-222 tested in this work contains free base porphyrins only. Motivated by the fact that a porphyrin-containing MOF, called compound 2, exhibits good adsorption capacity for methylene blue (MB), ${ }^{27}$ we tested the dye adsorption/removal ability of PCN-222 in this study.

Generally, MOFs are selective toward either cationic or anionic dyes. ${ }^{27-32}$ However, removing cationic and anionic dyes simultaneously is a challenge ${ }^{33}$ and mutual enhancement of individual adsorption by MOFs in a mixture of dye solution has never been reported. MB $(13.4 \times 5.0 \times 4.2 \AA)$ and MO $(14.5 \times$ $4.3 \times 3.1 \AA)$ are selected in this study as representative watersoluble cationic and anionic dyes, respectively, which are major-aromatic pollutants from textile, printing, paper and other industries. ${ }^{\mathbf{3 4}, 35}$ We tested the adsorption properties of PCN-222 toward each of them in single and combined dye systems. In addition, other cationic dyes, like malachite green (MG) and rhodamine $\mathrm{B}(\mathrm{RhB})$, as well as anionic dyes, like indigo carmine (IC), acid fuchsine (AF), acid orange (AO), and fluorescein disodium salt (FD), are also tested in this work.

\section{Experimental}

\section{Materials}

The raw materials used for PCN-222 synthesis and eight tested organic dyes were of analytical grade and were used without further purification. Methylene blue (MB, 98\% purity), rhodamine B (RhB, 98\%), and fluorescein disodium salt (FD, 98\%) were purchased from Innochem (Beijing, China). Malachite green (MG, 95\%), zirconyl chloride octahydrate $\left(\mathrm{ZrOCl}_{2} \cdot 8 \mathrm{H}_{2} \mathrm{O}\right.$, 99.9\%), and $\mathrm{KBr}$ (spectroscopic grade) were obtained from Aladdin (Shanghai, China). Methyl orange (MO, 98\%), indigo carmine (IC, 98\%), acid fuchsine (AF, 98\%), acid orange (AO, 97\%), and tetrakis (4-carboxypheneyl) porphyrin (TCPP, 97\%) were obtained from TCI (Tokyo, Japan). Sodium chloride ( $\mathrm{NaCl})$, $N, N$-dimethylformamide (DMF) and formic acid (98\%) were purchased from Sinopharm Chemical Reagent Co., Ltd. (Beijing, China). Acetone, methanol, hydrochloric acid ( $\mathrm{HCl}$ ), and sodium hydroxide $(\mathrm{NaOH})$ were purchased from Beijing Chemical Works (Beijing, China). Disposable chromatography columns were purchased from BIO-RAD (Hercules, USA). All aqueous solutions were prepared with deionized and purified water from a Milli-Q system.

\section{Instrumentation}

The morphology characterization was carried out on an optical microscope (Leica DMLM, Germany). Powder X-ray diffraction (PXRD) analysis was conducted on a D/max-2550 diffractometer (Rigaku, Japan) with $\mathrm{Cu}-\mathrm{K}_{\alpha}$ radiation $(\lambda=1.5418 \AA)$ for structure confirmation. To study the surface and porous properties, $\mathrm{N}_{2}$ adsorption/desorption analysis was performed on an ASAP 2020 Accelerated Surface Area and Porosimetry System from Micromeritics (Norcross, GA, USA) at $77 \mathrm{~K}$. The manufacturer's software was used for modelling Brunauer-Emmett-Teller (BET) surface area and to obtain the pore size distribution. Prior to the adsorption experiment, the materials were activated in DMF with $2 \mathrm{M} \mathrm{HCl}$, followed by washing with DMF and acetone several times. After soaking in acetone for a period of time, the sample was dried under vacuum for $6 \mathrm{~h}$ and then at $120{ }^{\circ} \mathrm{C}$ for $12 \mathrm{~h}$. The FT-IR experiment was conducted on an IFS 66v/S vacuum FT-IR instrument (Bruker, Germany) in the wavenumber range from 2000 to $400 \mathrm{~cm}^{-1}$, overlapped 32 times. The dye concentrations were measured by a UV-2700 spectrophotometer (Shimadzu, Japan).

\section{Preparation of PCN-222}

PCN-222 (MOF-545) was prepared by a solvothermal synthesis method according to a published protocol with slight modification. ${ }^{25}$ The zirconyl chloride octahydrate $(750 \mathrm{mg})$ was dissolved in $200 \mathrm{~mL}$ of DMF in a dry glass beaker by sonication. TCPP linker (130 mg) was added to the solution and then the mixture was sonicated again for another $10 \mathrm{~min}$. Finally, $140 \mathrm{~mL}$ of formic acid was added to the beaker. The final mixture was transferred into twenty $20 \mathrm{~mL}$ disposable scintillation vials equally. The vials were tightly closed and heated at $130{ }^{\circ} \mathrm{C}$ for $80 \mathrm{~h}$. The microcrystal precipitates in each vial were collected, combined and then washed with DMF 10 times or until the supernatant became clear. Afterwards, DMF was replaced with acetone to continue washing five times a day for 3 days. Finally, the acetone was carefully removed with a dropper and then the residual acetone was evaporated by placing the microcrystals in a thermostatic vacuum drier at $120^{\circ} \mathrm{C}$ for $2 \mathrm{~h}$. 


\section{Adsorption}

The effect of $\mathrm{pH}$ on $\mathrm{PCN}-222$ adsorption toward cationic MB, anionic MO and a mixture of them was examined. To avoid precipitate formation in the mixture, the initial dye concentration was limited to $10 \mathrm{mg} \mathrm{L}^{-1}$ of each, thus a large volume of dye solution was needed in order to reach adsorption saturation. The experiment was carried out in a $1 \mathrm{~L}$ sealed glass bottle with $5 \mathrm{mg}$ of PCN-222 by stirring mixing. The initial $\mathrm{pH}$ values of the dye solutions were adjusted using $\mathrm{HCl}(0.1 \mathrm{M})$ or $\mathrm{NaOH}(0.1 \mathrm{M})$. For adsorption kinetics and isotherm studies, a typical adsorption system contained $5 \mathrm{mg}$ of adsorbent in a $5 \mathrm{~mL}$ single dye solution in a disposable scintillation vial at an optimized $\mathrm{pH}$ and multiple dye concentrations ranging from 400 to $1000 \mathrm{mg} \mathrm{L}^{-1}$. The adsorbent was well mixed in the dye solution at room temperature by magnetic stirring on a MS-H280-Pro LED digital hotplate (SCILOGEX, USA). After the adsorption finished or at time $t$, the mixture or aliquot was centrifuged at $12000 \mathrm{rpm}$ for $30 \mathrm{~s}$. The absorbances of the dyes at their characteristic wavelengths in the supernatant were measured and compared with those before the adsorption. For MB and MO, the absorbance peaks were at 664 and $464 \mathrm{~nm}$, respectively. The adsorption amount $q_{t}\left(\mathrm{mg} \mathrm{g}^{-1}\right)$ at time $t(\mathrm{~min})$ and the adsorption capacity $q_{\mathrm{e}}\left(\mathrm{mg} \mathrm{g}^{-1}\right)$ at equilibrium were calculated as follows:

$$
\begin{aligned}
& q_{t}=\frac{\left(C_{0}-C_{t}\right) V}{m} \\
& q_{\mathrm{e}}=\frac{\left(C_{0}-C_{\mathrm{e}}\right) V}{m}
\end{aligned}
$$

where $C_{0}, C_{t}$ and $C_{\mathrm{e}}\left(\mathrm{mg} \mathrm{L}^{-1}\right)$ were the dye concentrations at the initial, any time $t$, and equilibrium in the solution, respectively. $V$ (L) was the volume of the dye solution and $m(\mathrm{~g})$ was the mass of PCN-222. $C_{0}$ was determined prior to the adsorption by measuring the amount of dye to be added. An adsorption spectrum of the initial dye solution was recorded at wavelength $664 \mathrm{~nm}$ to $464 \mathrm{~nm}$. A dilution may be needed for high concentration dye solutions to keep the absorbance within the range of the Beer-Lambert law. $C_{t}$ was calculated by comparing the characteristic absorbances of dyes before and at time $t$ after adsorption. $C_{\mathrm{e}}$ was the dye concentration in the supernatant at equilibrium time determined by comparing the characteristic absorbances of the dyes before and after adsorption. The method was also applied to adsorption kinetics, adsorption isotherms, and adsorption thermodynamics analysis. Two adsorption kinetic models, namely pseudo-first-order and pseudo-second-order, were used to evaluate the rate of adsorption of PCN-222 at three different concentrations. To determine the adsorption isotherms, Langmuir and Freundlich isotherm models were explored to study the adsorption mechanism and to obtain the theoretical maximum adsorption capacity. Furthermore, the thermodynamic parameters were calculated to ensure that either $\Delta H^{0}$ or $\Delta S^{0}$ promote the adsorption according to the Van't Hoff equation.

\section{Chromatography column adsorption}

A practical method for dye removal by a disposable chromatography column was introduced in this report. The device consisted of a column (20 mL) with upper and lower bed supports, end caps, and tip closures. The maximum column packing volume can be up to $10 \mathrm{~mL}$. A HL-2N constant flow pump (HuXi, China) was connected to the outlet of the column to adjust the flow rate. We tested eight anionic or cationic dyes and a mixture of $\mathrm{MB}$ and $\mathrm{MO}$ with an initial dye concentration of $10 \mathrm{mg} \mathrm{L}^{-1}$ and a flow rate of $2 \mathrm{~mL} \mathrm{~min}^{-1}$. An eluent $(0.1 \mathrm{M}$ $\mathrm{HCl} /$ methanol (1:9, v/v)) was employed for removal of $\mathrm{MB}, \mathrm{MG}$, RhB, MO, FD, and MB-MO, and another eluent (saturated $\mathrm{NaCl}$ / DMF $(1: 9, \mathrm{v} / \mathrm{v})$ ) was used for IC, AF, and AO. When the experiment was over, the column was washed and sealed with DMF for the next use.

\section{Results and discussions}

\section{Characterization and structure confirmation}

Fig. S1 $\uparrow$ shows optical microscopy images of PCN-222. The microcrystals of PCN-222 appear transparent and needleshaped within $100 \mu \mathrm{m}$ in length as prepared (S1a and S1b). PCN-222 turns to blue and purple after adsorbing MB (S1c) and MO (S1d), respectively. No significant morphology change was observed after the eight reuse cycles of adsorption/removal of MB (S1e) and MO (S1f). To confirm the microcrystalline structure, Fig. $\mathrm{S} 2 \uparrow$ displays PXRD patterns, which revealed four main diffraction peaks of PCN-222 at 2.26, 4.72, 7.12 and 9.76 degrees $(2 \theta)$, in agreement with previously published results. ${ }^{24}$ To assess the surface area and porous size of PCN-222, $\mathrm{N}_{2}$ adsorption/ desorption analyses are presented in Fig. S3. $\dagger$ The results show that the BET surface area is $2336 \mathrm{~m}^{2} \mathrm{~g}^{-1}$ and the mesoporous size is $32 \AA$, which are close to the published data of $2260 \mathrm{~m}^{2} \mathrm{~g}^{-1}$ for MOF-545 and $32 \AA$ for hexagonal meso-channels in PCN-222, respectively. ${ }^{24,25}$ We take advantage of the large pore feature of the PCN-222 to absorb and remove dyes with a wide range of particle sizes, as well as aggregates of anionic/cationic dyes, in a co-adsorption process in this study. Finally, the FT-IR results are presented in Fig. $\mathrm{S} 4$ and Table $\mathrm{S} 1, \dagger$ which agree with the fingerprint bands of the organic linker TCPP. ${ }^{36}$

\section{Effect of $\mathrm{pH}$ value on zeta potential, adsorption and co- adsorption of anionic/cationic dyes}

It is accepted that the key mechanism of adsorptive removal of dyes in aqueous solutions is electrostatic interactions between MOFs and dyes. ${ }^{17}$ Zeta potential is an adsorbent's characteristic that affects the interaction and the adsorption capacity. Fig. S5 $\dagger$ shows the zeta potentials of PCN-222 at a pH range of 3 to 10 , revealing the isoelectric point or point of zero charge (PZC) at $\mathrm{pH} 8$ with a potential range of $23.5 \mathrm{mV}$ at $\mathrm{pH} 3$ to $-13.6 \mathrm{mV}$ at $\mathrm{pH}$ 10. Because of the slight basic $\mathrm{pH}_{\mathrm{PzC}}$ and relatively large positive and negative potentials, we speculate that PCN-222 should be a stable absorbent toward both cationic and anionic dyes in solution. Using MO and MB as the representatives of anionic and cationic dyes, respectively, we examined the adsorption capacity of $\mathrm{PCN}-222$ over the $\mathrm{pH}$ range of 3-9 with an initial dye concentration of $10 \mathrm{mg} \mathrm{L}^{-1}$, as shown in Fig. 1. It was observed that the maximum adsorption for MO occurs at $\mathrm{pH} 5$ $\left(<\mathrm{pH}_{\mathrm{PZC}}\right.$, solid red circle), at which $\mathrm{MO}$ is preferentially in an 
anionic form, ${ }^{37}$ while PCN-222 has a positive zeta potential. However, a slight decrease of the adsorption capacity below $\mathrm{pH}$ 5 is noticed in Fig. 1 (solid red circles) indicating that a different absorptive mechanism may be secondarily involved in the process. On the contrary, at high $\mathrm{pH}$ values, the adsorption capacity toward cationic $\mathrm{MB}$ increases substantially in Fig. 1 (solid black squares). A quantitative comparison of the adsorption capacities of PCN-222 with other MOF absorbents is listed in Table 4. PCN-222 shows high efficacy in the table. Interestingly, when MO and MB co-exist in solution, PCN-222 demonstrates a mutual-beneficial enhancement in adsorption capacity, as shown in Fig. 1 (black open squares for MB and red open circles for MO). We found that the capabilities toward either MO or MB are greatly elevated across the tested $\mathrm{pH}$ range in the mixed dye solution. Particularly, at pH 9 MB enhances the adsorption of MO by $118 \%$ and vice versa, MO enhances MB by $96 \%$ at pH 5 compared with that in the signal dye system. In order to see the adsorption process in detail, Fig. 2a-c displays the UV-Vis spectra of the residual dyes of MB, MO, and MB-MO in the liquid phase at different times, respectively. The spectrum peaks at $664 \mathrm{~nm}$ and $464 \mathrm{~nm}$ are characteristic of MB and MO, respectively. In Fig. 2, a rapid decrease in the absorbance intensity within the first $5 \mathrm{~min}$ indicates the uptake time to be fast toward both anionic and cationic dyes. Finally, the absorbance of MB and MO decreases by 60 and 57\% for adsorbing individually and 78 and $70 \%$ for together, respectively, after $5 \mathrm{~h}$. The insets in Fig. 2 visually demonstrate the color change of the dyes in vials before and after the adsorption on PCN-222. We believe that the large pore size of PCN-222 facilitates the fast adsorption and makes the co-adsorption of large size MB-MO aggregates possible. Moreover, the suitable $\mathrm{pH}_{\mathrm{PzC}}$, as well as the relatively large positive/negative zeta potentials, explain the ability of PCN-222 to attract oppositely charged dyes.

\section{Adsorption kinetics}

To study the adsorption kinetics of PCN-222 toward MB and $\mathrm{MO}$, the effect of contact time on the adsorption capacity was

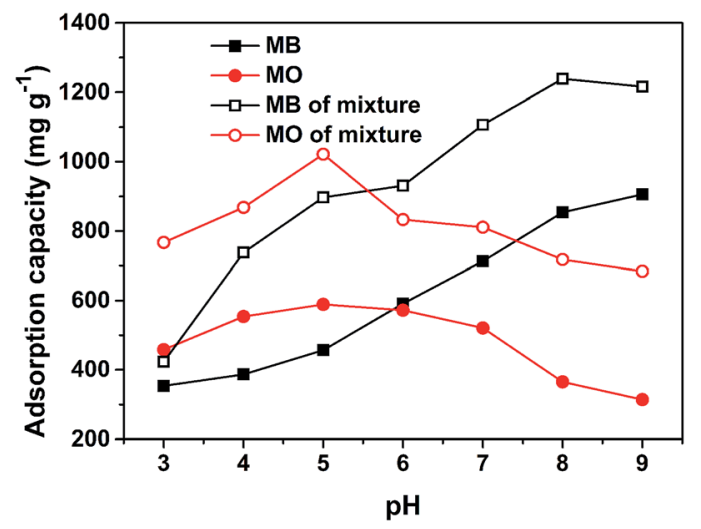

Fig. 1 Effect of initial $\mathrm{pH}$ on adsorption capacities of MB (black symbols) and $\mathrm{MO}$ (red symbols) in the single system (solid symbols) individually and in a mixed solution of MB-MO (open symbols) at $25^{\circ} \mathrm{C}$. The initial dye concentrations are $10 \mathrm{mg} \mathrm{L}^{-1}$ for each dye and the adsorption volume is $1 \mathrm{~L}$.
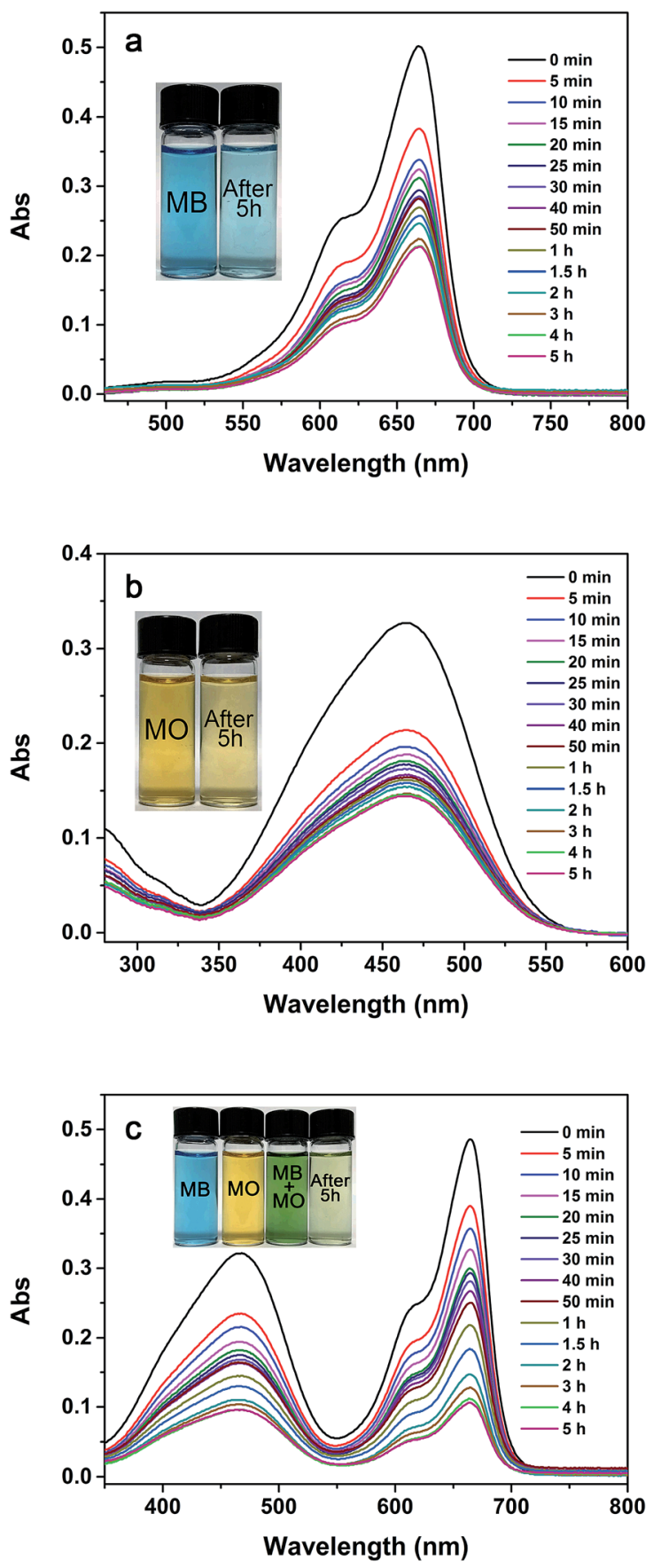

Fig. 2 UV-Vis spectra of (a) MB, (b) MO, and (c) mixture of MB-MO during adsorption at $\mathrm{pH} 6,25^{\circ} \mathrm{C}$, and different times in a $1 \mathrm{~L}$ solution containing $10 \mathrm{mg}$ of PCN-222 and $10 \mathrm{mg}$ of each dye. Each sample was diluted 2.5 times before UV-vis. The insets show the color changes before and $5 \mathrm{~h}$ after adsorption.

tested at room temperature using three levels of dye concentration $\left(1000,600\right.$, and $\left.400 \mathrm{mg} \mathrm{L}^{-1}\right)$. As described in the method, the absorbed amount is quantified from the absorbance difference prior to adsorption and at tested time $t$. According to the results in the previous section, the experimental $\mathrm{pH}$ is selected at the preferred values of $\mathrm{pH} 9$ and $\mathrm{pH} 5$ for $\mathrm{MB}$ and MO, respectively. As presented in Fig. $3 \mathrm{a}$ and b, the adsorption happens rapidly during the first $5 \mathrm{~min}$, then slows down in the 

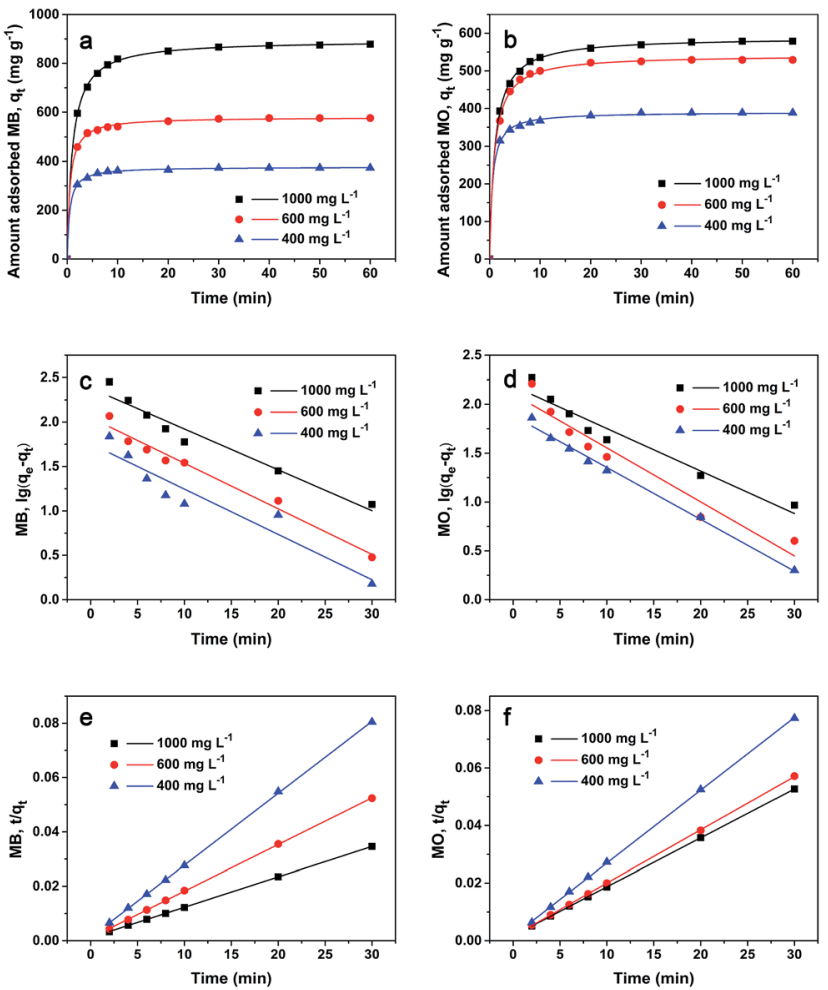

Fig. 3 Adsorption capacity of (a) MB and (b) MO as a function of time at different initial concentrations. Linear regressions of (c) MB and (d) $\mathrm{MO}$ according to pseudo-first-order kinetic model. Linear regressions of (e) MB and (f) MO according to pseudo-second-order kinetic model.

next $45 \mathrm{~min}$, and finally plateaus after $50 \mathrm{~min}$. Compared with the 3 day adsorption time by MIL-100(Fe), which is claimed to be a high capacity dye adsorbent, ${ }^{22}$ PCN-222 (32 A in pore size) takes a much shorter contact time to reach the maximum adsorption than MIL-100(Fe) (25 $\AA$, or $29 \AA$ ). This can be explained by PCN-222's large mesoporous structure and strong electrostatic interaction with MB and MO dyes. PCN-222 shows similar adsorption behavior toward $\mathrm{MB}$ and $\mathrm{MO}$, except concentration saturation occurs above $600 \mathrm{mg} \mathrm{L}^{-1}$ for the latter.

Two kinetic models, pseudo-first-order and pseudo-secondorder, are used to express the mechanism of $\mathrm{MB}$ and $\mathrm{MO}$ adsorption on PCN-222 at the three concentrations mentioned above. According to the pseudo-first order rate expression, ${ }^{38}$ the adsorption rate is based on the adsorption capacity; the linear equation in logarithms is as follows:

$$
\log \left(q_{\mathrm{e}}-q_{t}\right)=\log q_{\mathrm{e}}-\frac{k_{1}}{2.303} t
$$

where $q_{\mathrm{e}}$ and $q_{t}$ represent the amount of the dye adsorbed per unit PCN-222 $\left(\mathrm{mg} \mathrm{g}^{-1}\right)$ at equilibrium and at any time $t$, respectively, and $k_{1}\left(\mathrm{~min}^{-1}\right)$ is the pseudo-first-order rate constant, which can be obtained from the slope of the linear regression of eqn (3), as shown in Fig. 3c and d and Table 1. The experimental data do not seem to fit eqn (3) well, resulting in a correlation coefficient $R^{2}$ from 0.89299 to 0.97408 for MB and 0.92899 to 0.99189 for MO at different initial concentrations.
This indicates that the mechanism of the adsorption is not purely based on first-order kinetics.

We examined the adsorption data by a pseudo-second-order equation based on the rate controlling step being a chemisorption mechanism through sharing or exchanging the electrons between the dyes and PCN-222. The linear second-order equation is expressed as follows: ${ }^{39}$

$$
\frac{t}{q_{t}}=\frac{1}{k_{2} q_{\mathrm{e}}^{2}}+\frac{1}{q_{\mathrm{e}}} t
$$

where $k_{2}$ is the pseudo-second-order rate constant $\left(\mathrm{g} \mathrm{mg}^{-1}\right.$ $\min ^{-1}$ ). The calculated kinetic constants are shown in Table 1, and the fitting curves are shown in Fig. 3e and $\mathrm{f}$. The $R^{2}$ of the pseudo-second-order equation were from 0.99987 to 0.99996 and 0.99987 to 0.99998 for MB and MO, respectively, which were higher than those by pseudo-first-order model. The results indicate that the adsorption of $\mathrm{MB}$ and $\mathrm{MO}$ onto PCN-222 fits the pseudo-second-order kinetic model well.

\section{Adsorption isotherms}

At fixed $\mathrm{pH} 9$ for $\mathrm{MB}$ and $\mathrm{pH} 5$ for $\mathrm{MO}$, the adsorption isotherm studies were performed at three temperatures $(298,308$, and $318 \mathrm{~K})$ and seven initial dyes concentrations $(400,500,600,700$, 800,900 , and $1000 \mathrm{mg} \mathrm{L}^{-1}$ ). The experimental data were fitted to the Langmuir and Freundlich isotherm models to explore the adsorption mechanism..$^{\mathbf{4 0 , 4 1}}$

The Langmuir model is developed for the case of adsorbed molecules in aqueous solution onto solid adsorbents. The model assumes that a monolayer of adsorbate molecules covers a uniform surface of adsorbent homogeneously. It will take place on specific sites of the adsorbent only. The Langmuir equation in linear form is given as follows:

$$
\frac{C_{\mathrm{e}}}{q_{\mathrm{e}}}=\frac{1}{Q K_{\mathrm{L}}}+\frac{C_{\mathrm{e}}}{Q}
$$

where $C_{\mathrm{e}}$ is the equilibrium concentration of the adsorbate (mg $\left.\mathrm{L}^{-1}\right), Q$ is the maximum adsorption capacity $\left(\mathrm{mg} \mathrm{g}^{-1}\right)$, and $K_{\mathrm{L}}$ is the Langmuir constant related to the equilibrium of adsorption. The dimensionless separation factor $R_{\mathrm{L}}$ can be presented as follows:

$$
R_{\mathrm{L}}=\frac{1}{1+\left(1+K_{\mathrm{L}} C_{0}\right)}
$$

where $C_{0}$ is the initial concentration of the dye $\left(\mathrm{mg} \mathrm{L}^{-1}\right)$. When $0<R_{\mathrm{L}}<1$, the model is suggested to be favorable. On the other hand, the Freundlich model assumes that the adsorption takes place on heterogeneous surfaces, and the adsorption capacity is related to the dye concentration at equilibrium. ${ }^{42}$ The Freundlich equation is given as follows:

$$
\ln q_{\mathrm{e}}=\ln K_{\mathrm{F}}+\frac{1}{n} \ln C_{\mathrm{e}}
$$

where $K_{\mathrm{F}}$ is a Freundlich constant and $n$ is the heterogeneity factor related to the capacity and intensity of adsorption, respectively. As shown in Fig. 4 and Table 2, the adsorption of $\mathrm{MB}$ and MO onto PCN-222 fits the Langmuir model well over the entire studied concentration and temperature range for both 
Table 1 Adsorption kinetic parameters of $\mathrm{MB}$ and $\mathrm{MO}$ on $\mathrm{PCN}-222$

\begin{tabular}{|c|c|c|c|c|c|c|c|}
\hline Dyes & $C_{0}\left(\mathrm{mg} \mathrm{L}^{-1}\right)$ & \multicolumn{3}{|c|}{ Pseudo-first-order kinetics } & \multicolumn{3}{|c|}{ Pseudo-second-order kinetics } \\
\hline & 600 & 0.1183 & 112.4 & 0.97408 & $2.765 \times 10^{-3}$ & 581.4 & 0.99989 \\
\hline & 400 & 0.1175 & 57.02 & 0.89299 & $5.443 \times 10^{-3}$ & 377.4 & 0.99987 \\
\hline MO & 1000 & 0.1002 & 154.1 & 0.93595 & $1.661 \times 10^{-3}$ & 588.2 & 0.99998 \\
\hline
\end{tabular}
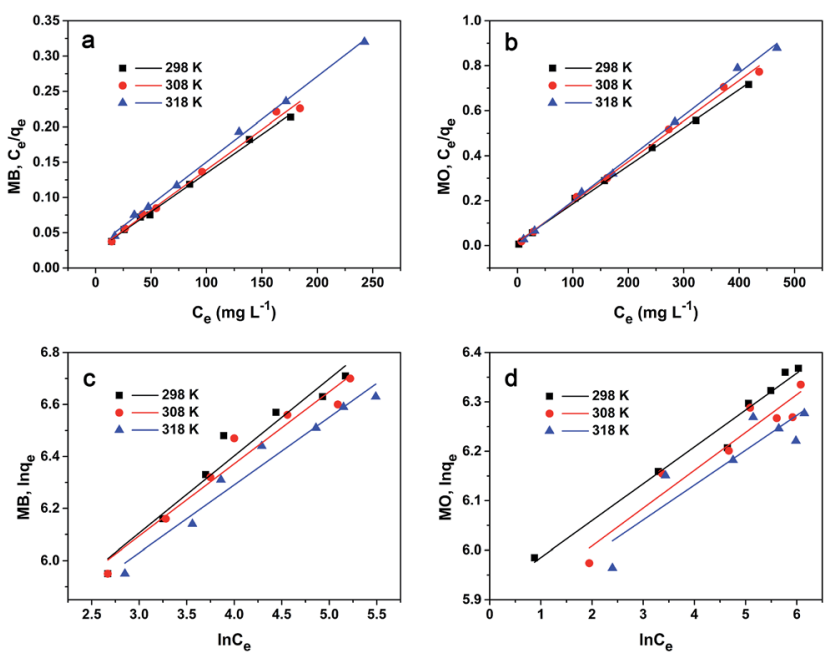

Fig. 4 Langmuir model of (a) MB and (b) MO adsorption onto PCN222. Freundlich model of (c) $M B$ and (d) $M O$ at three different temperatures.

dyes based on high linear correlation coefficient $R^{2}$ values. The maximum adsorption capacities $Q$ are $909.1 \mathrm{mg} \mathrm{g}^{-1}$ for $\mathrm{MB}$ and $591.7 \mathrm{mg} \mathrm{g}^{-1}$ for MO. The experimental adsorption capacities at different initial concentrations are shown in Fig. S6. $\dagger$

\section{Adsorption thermodynamics}

Thermodynamic parameters are employed to discuss the adsorption spontaneity. The adsorption data from the previous section were used. The changes in adsorption enthalpy $\left(\Delta H^{0}\right)$, entropy $\left(\Delta S^{0}\right)$ and Gibbs free energy $\left(\Delta G^{0}\right)$ were obtained from the following equations:

$$
\begin{gathered}
K_{0}=\frac{q_{\mathrm{e}}}{C_{\mathrm{e}}} \\
\Delta G^{0}=-R T \ln K_{0} \\
\ln K_{0}=-\frac{\Delta H^{0}}{R T}+\frac{\Delta S^{0}}{R}
\end{gathered}
$$

where $K_{0}$ is the thermodynamic equilibrium constant, $T$ is the temperature $(\mathrm{K})$, and $R$ is the gas constant $\left(8.314 \mathrm{~J} \mathrm{~mol}^{-1} \mathrm{~K}^{-1}\right)$. The plots of $\ln K_{0} v s .1 / T$ are presented in Fig. S7. $\dagger$ The thermodynamic parameters are listed in Table 3. The $\Delta G^{0}$ values of $\mathrm{MB}$ and MO are all negative, which means that the adsorption processes are spontaneous. The negative values of $\Delta H^{0}$ also indicate that the adsorption processes are exothermic and favorable for the adsorption of $\mathrm{MB}$ and $\mathrm{MO}$ on PCN-222. However, the negative values of $\Delta S^{0}$ suggest that the randomness decreased during the adsorption processes. As a consequence, the adsorption process would be controlled by an enthalpy effect rather than an entropy change.

\section{Comparison of PCN-222 adsorption capacity with selected MOFs}

Table 4 is a comparison of the adsorption capacity of PCN-222 toward MB and MO with representative MOFs reported previously. Those selected MOFs have high capacities, as shown in the list. However, it is obvious that most MOFs selectively adsorb either MB or MO on the top half of the table. For instance, the size-controlled MIL-100(Fe) and complex 2, a porphyrin-containing MOF, strongly adsorb MB rather than MO. ${ }^{22,27}$ Conversely, 1Y, a Mn ${ }^{\mathrm{II}}$ phosphonate MOF, has strong affinity to MO, but it can hardly remove $\mathrm{MB}$ from the

Table 2 Isotherm parameters of MB and MO adsorption onto PCN-222

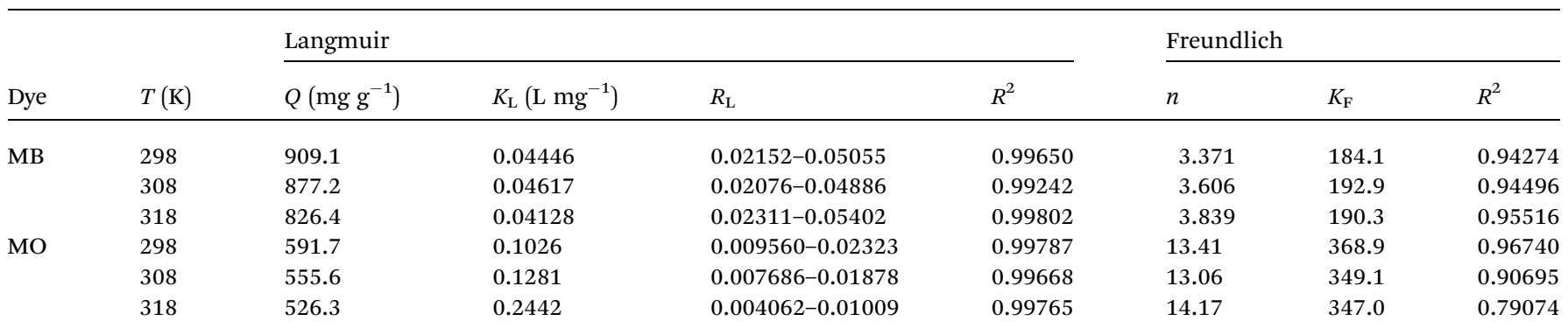


Table 3 Thermodynamic parameters of $\mathrm{MB}$ and $\mathrm{MO}$ adsorption onto $\mathrm{PCN}-222$

\begin{tabular}{llllll}
\hline Dye & $T(\mathrm{~K})$ & $\ln K_{0}$ & $\begin{array}{l}\Delta G^{0} \\
\left(\mathrm{~kJ} \mathrm{~mol}^{-1}\right)\end{array}$ & $\begin{array}{l}\Delta H^{0} \\
\left(\mathrm{~kJ} \mathrm{~mol}^{-1}\right)\end{array}$ & $\begin{array}{l}\Delta S^{0} \\
\left(\mathrm{~J} \mathrm{~mol} \mathrm{~mol}^{-1} \mathrm{~K}^{-1}\right)\end{array}$ \\
\hline \multirow{2}{*}{ MB } & 298 & 1.544 & -3.825 & -15.93 & -40.55 \\
& 308 & 1.365 & -3.495 & & \\
\multirow{4}{*}{ MO } & 2918 & 1.139 & -3.011 & & -24.47 \\
& 298 & 0.3351 & -0.8302 & -8.136 & \\
& 308 & 0.2474 & -0.6355 & & \\
& 318 & 0.1282 & -0.3389 & & \\
\end{tabular}

solution. ${ }^{43}$ Among the dyes with dual capabilities, MIL-100(Fe) ranks at the top, which adsorbs 736.2 and $1045 \mathrm{mg} \mathrm{g}^{-1}$ of $\mathrm{MB}$ and $\mathrm{MO}$, respectively, but the adsorption takes 3 days to reach the maximum. ${ }^{22}$ However, PCN-222 is able not only to remove MB and MO efficiently from a single dye solution or a mixture but also to remove them within only $60 \mathrm{~min}$. Actually, about $60 \%$ of MB or $39 \%$ of MO can be removed by PCN-222 within 2 min. Furthermore, the adsorption capacity of PCN-222 greatly increases to 1239 and $1022 \mathrm{mg} \mathrm{g}^{-1}$ for MB and MO, respectively, in the mixture. In addition, the synthesis of PCN222 is easily controlled. Good solvent stability and reusability are further advantages. PCN-222 indeed is a promising adsorbent for removing cationic and anionic dyes from aqueous solutions.

\section{Compact chromatography column for dye removal}

The above results indicate that PCN-222 is a promising potential adsorbent for removing dyes in solution. However, removal of dyes in disposable scintillation vials by centrifuging is not

Table 4 The adsorption capacity of selected MOFs and PCN-222 toward $\mathrm{MB}$ and $\mathrm{MO}$

\begin{tabular}{|c|c|c|c|c|}
\hline \multirow[b]{2}{*}{ MOF } & \multicolumn{2}{|c|}{$\begin{array}{l}\text { Adsorption capacity } \\
\left(\mathrm{mg} \mathrm{g}^{-1}\right)\end{array}$} & \multirow[b]{2}{*}{ Organic linker } & \multirow[b]{2}{*}{ Ref. } \\
\hline & MB & MO & & \\
\hline $\begin{array}{l}\text { Size-controlled } \\
\text { MIL-100(Fe) }\end{array}$ & 1105 & NA & BTC & 44 \\
\hline Complex 2 & 952 & NA & DCPP & 27 \\
\hline ZJU-24 & 902 & NA & TPTC/TPHC & 28 \\
\hline $\begin{array}{l}\text { DUT-23(Cu) } \\
\text { nanoparticle }\end{array}$ & 814 & NA & BTB/BPY & 23 \\
\hline Compound 2 & 725 & NA & TATAT & 45 \\
\hline $1 \mathrm{Y}$ & NA & 1337 & $\mathrm{H}_{4} \mathrm{~L} / \mathrm{bibp}$ & 43 \\
\hline TMU-16- $\mathrm{NH}_{2}$ & NA & 393.7 & $\begin{array}{l}\mathrm{NH}_{2}-\mathrm{BDC} / \\
\text { 4-bpdh }\end{array}$ & 46 \\
\hline PED-MIL-101 & NA & 194 & 1,4-BDC & 30 \\
\hline MIL-100(Fe) & 736.2 & 1045 & 1,3,5-ВTC & 22 \\
\hline PCN-222 & 906 & 589 & TCPP & This work \\
\hline PCN-222 & $\begin{array}{l}1239 \\
(\mathrm{MB}-\mathrm{MO})\end{array}$ & $\begin{array}{l}1022 \\
(\mathrm{MB}-\mathrm{MO})\end{array}$ & TCPP & This work \\
\hline $\mathrm{NH}_{2}$-MIL-101(Al) & 762 & 188 & 1,4-BDC & 47 \\
\hline MIL-100(Cr) & 643.3 & 211.8 & 1,3,5-BTC & 22 \\
\hline MOF-235 & 252.0 & 477.0 & 1,4-BDC/DMF & 21 \\
\hline
\end{tabular}

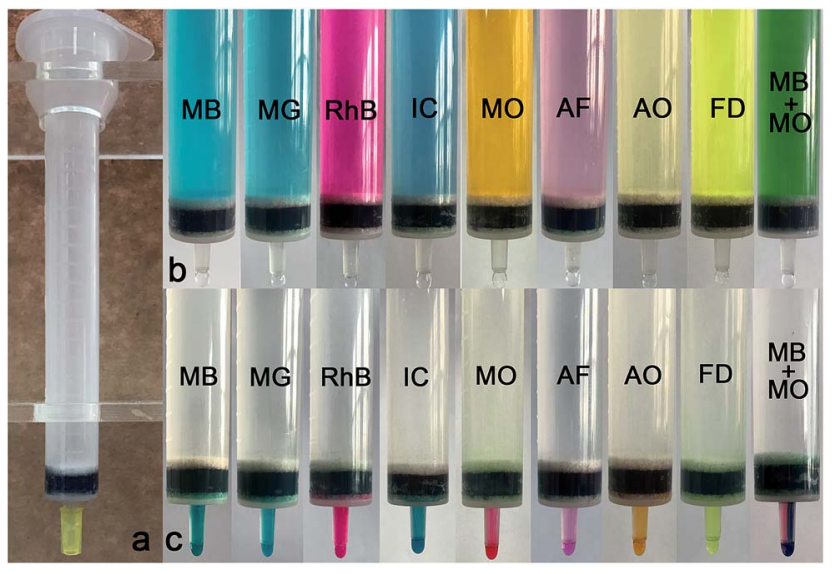

Fig. 5 (a) Chromatography column is filled with $\mathrm{PCN}-222$ microcrystalline $200 \mathrm{mg}$. (b) Eight cationic or anionic dyes or MB-MO mixture are loaded on the column. (c) Dyes are washed off by $0.1 \mathrm{M}$ $\mathrm{HCl} /$ methanol or saturated $\mathrm{NaCl} / \mathrm{DMF}$.

practicable in real applications. We therefore made a chromatography column using PCN-222 crystals, as shown in Fig. 5a. These disposable biological chromatography columns are easy to handle and reuse. The volume of the filler can be customized as required. Cationic or anionic dyes including $\mathrm{MB}, \mathrm{MG}, \mathrm{RhB}, \mathrm{MO}$, IC, AF, AO, FD and MB-MO mixture at a concentration of $10 \mathrm{mg}$ $\mathrm{L}^{-1}$ are applied to the column by pumping the liquid out from the outlets of the columns. The top photograph in Fig. 5b shows eight dyes and one mixture loaded into the columns, and the effluent is colorless for all. As the maximum adsorption is reached when the effluent starts to show color, the pumping is stopped. The dyes can also be immediately washed off using organic eluent $(0.1 \mathrm{M} \mathrm{HCl} / \mathrm{methanol}$ at $1: 9 \mathrm{v} / \mathrm{v})$ for $\mathrm{MB}, \mathrm{MG}, \mathrm{RhB}$, MO, FD, MB-MO or saturated NaCl/DMF (1: $9 \mathrm{v} / \mathrm{v})$ for IC, AF, AO. The color effluent can be monitored not only by the naked eye but also by UV-Vis adsorption, as shown in the bottom photograph in Fig. 5c. The same column was used during the entire chromatography column experiment, offering a perspective on the stability and reusability of PCN-222. The maximum adsorption capacities of PCN-222 toward different dyes at pH 6 are listed in Table S2.†

\section{The reusability of $\mathrm{PCN}-222$ in column}

The reusability of adsorbents is vital in dye removal application. Most adsorbents are not utilized repeatedly because of difficult desorption. In this work, $\mathrm{MB}$ or MO desorption from PCN-222 was conducted using $0.1 \mathrm{M} \mathrm{HCl} / \mathrm{methanol}(1: 9, \mathrm{v} / \mathrm{v})$ and the entire adsorption/desorption was performed on the column with $5 \mathrm{mg}$ PCN-222 filling. In this work, sufficient volumes of $\mathrm{MB}$ or $\mathrm{MO}\left(10 \mathrm{mg} \mathrm{\textrm {L } ^ { - 1 }}\right)$ to give adsorption saturation were loaded. We define the adsorption capacity in the first cycle as $100 \%$. The data in Fig. 6 indicates the removal efficiency only decreases to $95.2 \%$ for $\mathrm{MB}$ and to $92.7 \%$ for $\mathrm{MO}$ after eight reuse cycles. Furthermore, neither morphology nor structure changes of PCN-222 are observed after eight cycles, as shown in Fig. S1 and $\mathrm{S} 2 . \dagger$ The results above indicate the reusability of PCN-222. 


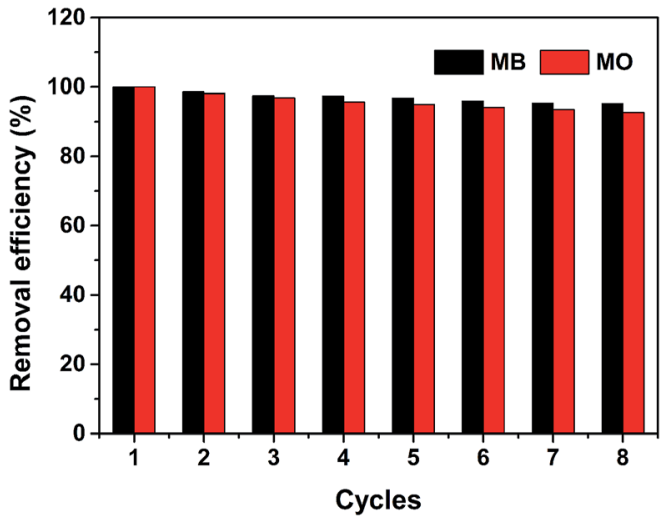

Fig. 6 Reusability test of PCN-222 for removal of $\mathrm{MB}$ and $\mathrm{MO}$. The first cycle is defined as $100 \%$ at maximum capacity for each dye at $\mathrm{pH} 6$. Black bars are for $\mathrm{MB}$ and red for $\mathrm{MO}$. The loading dye concentration is $10 \mathrm{mg} \mathrm{L}^{-1}$.

\section{Proposed mechanism of mutual enhancement in co- adsorption system}

PCN-222 consists of $\mathrm{Zr}_{6}$ clusters coordinated to free base TCPPs and terminal hydroxyl groups in the shape of a 1D channel with an octagon in the cross section. ${ }^{24}$ At low $\mathrm{pH}$, the hydroxyl groups fall off and $\mathrm{Zr}_{6}$ clusters possess positive charges. At high $\mathrm{pH}$, the centers of the free base TCPPs carry negative charges, which makes PCN-222 able to interact with anionic MO and cationic MB. Interestingly, the results in the early section show an elevation of the adsorption capacity of both MB and MO in the mixture solution of $10 \mathrm{mg} \mathrm{L^{-1 }}(\approx 31 \mu \mathrm{M})$ of each across the tested $\mathrm{pH}$ range (Fig. 1). A push-pull mechanism may explain this mutual enhancement. We propose that MO can push MB dimerization and the MB dimer can pull MO together to adsorb on PCN-222, and vice versa. Previously published articles support this hypothesis. First of all, it is known that most organic dyes in solution are in the forms of dimers, trimers and higher aggregates through self-aggregation depending on the concentrations. ${ }^{48}$ In the same way, planar molecules of MB can form face-to-face dimers in solution by self-association through $\pi-\pi$ stacking interactions between aromatic rings, as shown in Fig. 7.49,50 The monomer-dimer equilibrium is observed at concentrations of 2 to $50 \mu \mathrm{M}$. $^{51}$ Then the MB dimer carries a positive charge at each end (Fig. 7) because of the repulsion force between them. We expect that one end of the dimer interacts with the center of the free base TCPP, and the other end pulls anionic MO, as shown in Fig. 7. Hence, the adsorption capacity of MO increases. However, with the dimer dissociation constant of $2.9 \times 10^{-4} \mathrm{M}$ in water, ${ }^{50}$ only $10 \% \mathrm{MB}$ dimer may form at $10 \mathrm{mg} \mathrm{L}^{-1}$ dye concentration. To explain $118 \%$ enhancement of MO's adsorption capacity in the co-adsorption system at $\mathrm{pH} 9$, it is speculated that MO may stimulate MB dimerization. Interestingly, it has been reported that when adding anionic sodium dodecyl sulfate, $\mathrm{MB}$ aggregation increases more than 20 fold. ${ }^{51}$ Thus, cationic MB's dimerization could be promoted in the presence of anionic MO. In fact, we have observed that precipitates form when each dye concentration is above $20 \mathrm{mg} \mathrm{L}^{-1}$ in the mixture solution. In turn, like

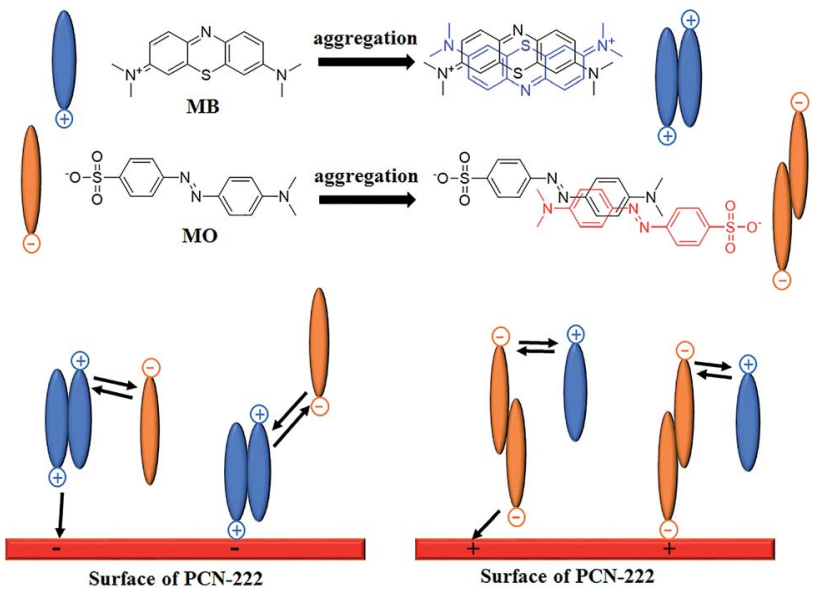

Fig. 7 Schematic diagram of the push-pull mechanism.

MB, we speculate that MO can also form dimers (Fig. 7) that can pull more $\mathrm{MB} .{ }^{52}$ Indeed, it has been reported that MO is almost completely dimerized above $0.2 \mathrm{mM}$ and has an estimated dimer formation constant $K_{\mathrm{d}}$ of $9200,{ }^{53}$ which means that $27 \%$ of $\mathrm{MO}$ is in dimer form at $10 \mathrm{mg} \mathrm{L}^{-1}$. One end of the dimer of MO is linked with a $\mathrm{Zr}_{6}$ cluster, and the other end attracts a $\mathrm{MB}$ molecule. Finally, a diagram to explain the mechanism is shown in Fig. 7. Furthermore, the large mesopore size of PCN-222 makes aggregate adsorption by the push-pull mechanism possible.

\section{Conclusions}

In summary, the features of large pore size and suitable zeta potentials of PCN-222, a zirconium-metalloporphyrin mesoMOF, facilitate the high adsorption capacity for cationic and anionic dyes on the absorbent in aqueous solution. The large surface area of PCN-222 makes rapid adsorption and easy desorption possible. More significantly, the mutual enhancement of adsorption capacity in a mixture dye solution is observed and explained by a push-pull mechanism. The phenomenon has never been reported before in MOFs and can provide insight into improving dye adsorption/removal efficacy. Two representative dyes (cationic MB and anionic MO) are used to investigate the adsorption kinetics, isotherms, and thermodynamics of $\mathrm{MB}$ and MO on PCN-222. The kinetic data fits the pseudo-second-order kinetic model well. According to the best-fit Langmuir model, the adsorption is a monolayer coverage process. The adsorption is also exothermic and is controlled by an enthalpy effect. The excellent adsorption properties of PCN-222 motivated us to make a chromatography column for practical applications. Furthermore, the PCN-222 also possesses good reusability, which is crucial for field use of wastewater treatment.

\section{Acknowledgements}

The authors are grateful for the financial support from the National Natural Science Foundation of China (Grant Numbers: 31670797). 


\section{Notes and references}

1 G. Crini, Bioresour. Technol., 2006, 97, 1061-1085.

2 R. D. Ambashta and M. Sillanpää, J. Hazard. Mater., 2010, 180, 38-49.

3 F. Liu, S. Chung, G. Oh and T. S. Seo, ACS Appl. Mater. Interfaces, 2012, 4, 922-927.

4 P. B. S. Ratna, Int. J. Environ. Sci., 2012, 3, 940-955.

5 D. Parasuraman and M. J. Serpe, ACS Appl. Mater. Interfaces, 2011, 3, 2732-2737.

6 M. M. Hamed, I. M. Ahmed and S. S. Metwally, J. Ind. Eng. Chem., 2014, 20, 2370-2377.

7 V. K. Gupta and T. A. Saleh, Environ. Sci. Pollut. Res., 2013, 20, 2828-2843.

8 A. A. Adeyemo, I. O. Adeoye and O. S. Bello, Toxicol. Environ. Chem., 2012, 94, 1846-1863.

9 L. C. D. S. Maria, M. R. M. P. Aguiar, P. D'Elia, L. O. Ferreira and S. H. Wang, Mater. Lett., 2007, 61, 3395-3399.

10 V. Simon, A. Thuret, L. Candy, S. Bassil, S. Duthen, C. Raynaud and A. Masseron, Chem. Eng. J., 2015, 280, 748-754.

11 N. Öztekin, A. Alemdar, N. Güngör and F. B. Erim, Mater. Lett., 2002, 55, 73-76.

12 X. Zhuang, Y. Wan, C. Feng, Y. Shen and D. Zhao, Chem. Mater., 2009, 21, 706-716.

13 W. Yu, L. Deng, Y. Peng, L. Dong, W. Yuan, L. Peng, H. He, Z. Li and F. Chen, J. Colloid Interface Sci., 2015, 448, 545-552.

14 J. Ma, F. Yu, L. Zhou, L. Jin, M. Yang, J. Luan, Y. Tang, H. Fan, Z. Yuan and J. Chen, ACS Appl. Mater. Interfaces, 2012, 4, 5749-5760.

15 J. Yin, M. Pei, Y. He, Y. Du, W. Guo and L. Wang, RSC Adv., 2015, 5, 89839-89847.

16 J. N. Sahu, J. Acharya and B. C. Meikap, J. Hazard. Mater., 2009, 172, 818-825.

17 (a) Z. Hasan and S. H. Jhung, J. Hazard. Mater., 2015, 283, 329-339; (b) Y. X. Tian, Y. P. He, M. Wang and J. Zhang, RSC Adv., 2014, 4, 1480-1483; (c) Y. P. He, Y. X. Tian and J. Zhang, Acta Chim. Sin., 2014, 72, 1228.

18 C. Wang, X. Liu, D. N. Keser, J. P. Chen and K. Li, Chem. Soc. Rev., 2016, 45, 5107-5134.

19 K. K. Gangu, S. Maddila, S. B. Mukkamala and S. B. Jonnalagadda, Inorg. Chim. Acta, 2016, 446, 61-74.

20 E. Haque, J. E. Lee, I. T. Jang, Y. K. Hwang, J. S. Chang, J. Jegal and S. H. Jhung, J. Hazard. Mater., 2010, 181, 535-542.

21 E. Haque, J. W. Jun and S. H. Jhung, J. Hazard. Mater., 2011, 185, 507-511.

22 M. Tong, D. Liu, Q. Yang, S. Devautourvinot, G. Maurin and C. Zhong, J. Mater. Chem. A, 2013, 1, 8534-8537.

23 Z. P. Qi, J. M. Yang, Y. S. Kang, F. Guo and W. Y. Sun, Dalton Trans., 2016, 45, 8753-8759.

24 D. Feng, Z. Y. Gu, J. R. Li, H. L. Jiang, Z. Wei and H. C. Zhou, Angew. Chem., 2012, 51, 10307-10310.

25 W. Morris, B. Volosskiy, S. Demir, F. Gándara, P. L. Mcgrier, H. Furukawa, D. Cascio, J. F. Stoddart and O. M. Yaghi, Inorg. Chem., 2012, 51, 6443-6445.
26 Y. Z. Chen and H. L. Jiang, Chem. Mater., 2016, 28, 66986704.

27 Y. Hou, J. Sun, D. Zhang, D. Qi and J. Jiang, Chem.-Eur. J., 2016, 22, 6345-6352.

28 Q. Zhang, J. Yu, J. Cai, R. Song, Y. Cui, Y. Yang, B. Chen and G. Qian, Chem. Commun., 2014, 50, 14455-14458.

29 X. Liu, W. Gong, J. Luo, C. Zou, Y. Yang and S. Yang, Appl. Surf. Sci., 2016, 362, 517-524.

30 E. Haque, J. E. Lee, I. T. Jang, Y. K. Hwang, J. S. Chang, J. Jegal and S. H. Jhung, J. Hazard. Mater., 2010, 181, 535542.

31 T. Wang, P. Zhao, N. Lu, H. Chen, C. Zhang and X. Hou, Chem. Eng. J., 2016, 295, 403-413.

32 L. Liu, J. Ge, L. T. Yang, X. Jiang and L. G. Qiu, J. Porous Mater., 2016, 23, 1-10.

33 A. Ayati, M. N. Shahrak, B. Tanhaei and M. Sillanpää, Chemosphere, 2016, 160, 30-44.

34 M. Rafatullah, O. Sulaiman, R. Hashim and A. Ahmad, J. Hazard. Mater., 2010, 177, 70-80.

35 A. Mittal, A. Malviya, D. Kaur, J. Mittal and L. Kurup, J. Hazard. Mater., 2007, 148, 229-240.

36 J. Wan, H. Wang, Z. Wu, Y. C. Shun, X. Zheng and D. L. Phillips, Phys. Chem. Chem. Phys., 2011, 13, 1018310190.

37 H. Liu, L. Chen and J. Ding, RSC Adv., 2016, 6, 48884-48895.

38 Y. S. Ho and G. Mckay, Process Saf. Environ. Prot., 1998, 76, 332-340.

39 M. I. El-Khaiary, G. F. Malash and Y. S. Ho, Desalination, 2010, 257, 93-101.

40 I. Langmuir, J. Am. Chem. Soc., 1918, 143, 1361-1403.

41 H. M. F. Freundlich, J. Am. Chem. Soc., 1906, 57, 385.

42 G. P. Hao, W. C. Li, S. Wang, S. Zhang and A. H. Lu, Carbon, 2010, 48, 3330-3339.

43 C. Y. Gao, Y. Yang, J. Ai, H. R. Tian, L. J. Li, W. Yang, S. Dang and Z. M. Sun, Chem.-Eur. J., 2016, 22, 11652-11659.

44 F. Tan, L. Min, K. Li, Y. Wang, J. Wang, X. Guo, G. Zhang and C. Song, Chem. Eng. J., 2015, 281, 360-367.

45 Z. Zhu, Y. L. Bai, L. Zhang, D. Sun, J. Fang and S. Zhu, Chem. Commun., 2014, 50, 14674-14677.

46 M. Roushani, Z. Saedi and T. M. Beygi, J. Taiwan Inst. Chem. Eng., 2016, 66, 164-171.

47 E. Haque, V. Lo, A. Minett, A. Harris and T. Church, J. Mater. Chem. A, 2013, 2, 193-203.

48 N. Florence and H. Naorem, J. Mol. Liq., 2014, 198, 255-258. 49 D. Heger, J. Jirkovský and P. Klán, J. Phys. Chem. A, 2005, 109, 6702-6709.

50 K. Patil, R. Pawar and P. Talap, Phys. Chem. Chem. Phys., 2000, 2, 4313-4317.

51 E. Morgounova, Q. Shao, B. J. Hackel, D. D. Thomas and S. Ashkenazi, J. Biomed. Opt., 2013, 18, 56004.

52 R. V. D. Coevering, P. C. A. Bruijnincx, M. Lutz, A. L. Spek, G. V. Koten and R. J. M. K. Gebbink, New J. Chem., 2007, 31, 1337-1348.

53 K. L. Kendrick and W. R. Gilkerson, J. Solution Chem., 1987, 16, 257-267. 